\title{
Perioperative antibiotic prescribing in two private sector hospitals in central India
}

\author{
J Sparrentoft ${ }^{*}$ M Sharma \\ From 3rd International Conference on Prevention and Infection Control (ICPIC 2015) \\ Geneva, Switzerland. 16-19 June 2015
}

\begin{abstract}
Introduction
Antibiotic resistance is increasing globally and high use of antibiotics has been found to correlate with increasing antibiotic resistance. Surgery related prophylactic use of antibiotics is common due to the high risk for patients to develop surgical site infections. In India, the world's second most populated country there is currently no national prescribing guidelines to be implemented and data, both on overall and prophylactic use antibiotics in surgery is scarce.
\end{abstract}

\section{Objectives}

To analyse the perioperative antibiotic prescribing among patients admitted with surgery indications at surgery departments of two tertiary care hospitals, a teaching hospital $(\mathrm{TH})$ and a non-teaching $(\mathrm{NTH})$.

\section{Methods}

The study was conducted in Ujjain, Madhya Pradesh, India. Patients admitted between April 2008 and March 2011 at the surgery wards of the CR. Gardi Hospital (TH) and Ujjain Charitable Trust Hospital (NTH) were included in the study, 6171 from the TH and 6263 from the NTH. Four diagnosis groups were devised, upper/ lower urinary tract and routine/emergency abdominal surgery indications. Patients with simultaneous infections were excluded. Demographic information and antibiotic prescribing data were analysed. DDDs were used to compare dosages. Adherence to the WHO list of essential medicines (WHOLEM) and National list of essential medicines of India (NLEMI) were analysed. antibiotics were imidazole derivates and quinolones at the $\mathrm{TH}$ and cephalosporins and antibiotic fixed dose combinations at the NTH. The average duration of hospital stay and duration of antibiotic treatment were 10.0 and 7.6 days at the TH and 3.9 and 3.2 days at the NTH. The overall average DDD/100 patient days were 72.5 in the TH and 110.5 at the NTH. Twenty five percent of prescriptions at the $\mathrm{TH}$ and $6 \%$ at the NTH were done using generic names. Adherence to WHOLEM and NLEMI were seen in $66 \%$ and $75 \%$ of prescriptions at the $\mathrm{TH}$ and $42 \%$ and $57 \%$ of prescriptions at the NTH.

\section{Conclusion}

The result from the study indicates that a large proportion of patients admitted at surgery wards of the study hospitals were prescribed prophylactic antibiotics. It also indicates that the antibiotics are being prescribed in long courses and that there are large differences in the dosages prescribed.

\section{Disclosure of interest}

None declared.

Published: 16 June 2015

doi:10.1186/2047-2994-4-S1-P176

Cite this article as: Sparrentoft and Sharma: Perioperative antibiotic

prescribing in two private sector hospitals in central India. Antimicrobial Resistance and Infection Control 2015 4(Suppl 1):P176.

\section{Results}

At the TH $88 \%$ and at the NTH $86 \%$ of patients were prescribed antibiotics. The most commonly prescribed

Department of Public Health Sciences, Karolinska Institutet, Sweden 